\title{
Exploration and Research on the Teaching of Operations Research Experimental Basis on MOOCs
}

\author{
Yazheng Dang1* ${ }^{*}$ Zhonghui Xue ${ }^{2}$ \\ ${ }^{1}$ School of Management, University of Shanghai for Science and Technology, Shanghai, China \\ ${ }^{2}$ Department of Basic Teaching, Shanghai publishing and Printing College, Shanghai, China \\ Email: *jgdyz@163.com
}

How to cite this paper: Dang, Y.Z. and Xue, Z.H. (2020) Exploration and Research on the Teaching of Operations Research Experimental Basis on MOOCs. Open Access Library Journal, 7: e6001.

https://doi.org/10.4236/oalib.1106001

Received: December 13, 2019

Accepted: January 5, 2020

Published: January 8, 2020

Copyright $\odot 2020$ by author(s) and Open Access Library Inc.

This work is licensed under the Creative

Commons Attribution International

License (CC BY 4.0).

http://creativecommons.org/licenses/by/4.0/

(c) (i) Open Access

\begin{abstract}
The MOOCs class and operational research teaching combination in this paper, through the analysis of the characteristics of the MOOCs class, operations research experiment course features and traditional logistics problems and challenges faced by the teaching, study MOOCS class under the background of operational research teaching mode construction, transforming teaching teacher role, and promote the fusion of MOOCs class and the teaching of operations research experimental.
\end{abstract}

\section{Subject Areas}

Education

\section{Keywords}

MOOCs, Operations Research Experimental, Teaching Reform

\section{1. 引言}

当前, 随着国家倡导建立慕课教育教学平台工作的深入, 传统的运筹学 实验教学面临着新的要求和挑战。运筹学是用定性与定量分析结合的方法来 研究现实世界系统运行的规律, 从中提出具有共性的模型, 寻求解决模型的 算法, 帮助决策者选择最优的决策方案, 以达到人力, 物力和财力的经济有 效配置。其广泛应用在生产管理, 工程技术等方面, 也越来越受到各部门和 企业的高度重视。但是一方面由于传统的运筹学教学仅局限于院校内部, 优 质运筹学教学资源多局限于一个院校内部, 受益者多局限在学校内部, 这样 运筹学爱好者, 企业和社会各部门会被限制在课堂之外; 另一方面院校内的 
运筹学实验教学多是配合课堂教学辅助课, 运筹学实验学时少, 课时大多限 制在 4 8 学时, 这些情况与运筹学课程具有的理论性, 实践性以及应用性特 点相矛盾。慕课的出现为解决以上的矛盾提供了一个全新的途径, 也为运筹 学实验教学质量的提升, 学生学习能力和应用能力的提升提供了一个可以实 现的平台, 为企业决策者提供了决策依据。作为多年从事运筹学课堂教学, 实验教学和科研工作的一线教师, 我们将试图利用慕课在线课堂的优势来解 决运筹学实验教学面临的问题, 并以此为契机推动慕课背景下的运筹学实验 混合式教学的新篇章, 适应运筹学教学的发展趋势, 实现传统课堂和在线课 堂的相互补充。

\section{2. 慕课及其特点}

大规模在线公开课程(MOOCs, 简称慕课)起源于 2012 年, 斯坦福大学两 位教授创立了 Coursera 在线免费课程平台, 目前已有 500 多门优质慕课在普 林斯顿、斯坦福大学等 100 余所世界一流大学开启, 后来, 麻省理工学院和 哈佛大学联手发布 edX 网络在线教学计划, 《电子和电路》作为第一门慕课, 开启一年已经 12 万名学生的注册, 目前上线学习人数已超过 120 万[1]。

与传统视频教学和网络公开课不同, 慕课能够将世界范围内的授课者和 学习者通过线上虚拟教室联系在一起。总的来说, 慕课有三个明显的特点, 一是规模庞大(Massive), 慕课的学习者来自世界各地, 只要有网络, 那么受 益范围就可无限延伸; 二是授课方式灵活多变, 授课者不再局限于传统课堂 上的演示文稿展示和乏味的语言描述，其可以通过微视频、微动画、温馨提 示、随堂演练向学习者传授知识, 最大程度地激发学习者的能动性; 三是互 动性极大地提高, 学习者可以通过聊天界面随时与授课者或其他学习者探讨 问题, 形成大规模的学习论坛, 在一定程度上能够促进师生之间以及学习者 之间的交流互动。

\section{3. 传统运筹学实验教学面临的问题}

作为理论运筹学教学的辅助课, 传统运筹学实验教学面临的主要问题有 三类:

3.1. 学时少、教学地域固化、人员有限。目前传统的运筹学实验教学只 能在各类学校中以一些指定场所开展传统的运筹学实验教学中, 由于教室容 量及计算机系统的限制, 导致参与学习的学生数量有限, 也使得广大运筹学 爱好者被拒于高校课堂之外[2]。

3.2. 授课过程多采用固定的案例, 不能对接新形式下出现的新问题; 老 师在课堂中扮演着主要角色, 教师根据自己的教学目标, 制定相应的教学大 纲, 课堂上以完成教学计划为主要目的, 学生机械式地接受, 机械地模仿, 缺乏对学生独立思考、解决实际案例的能力的训练, 也忽略了学生创新能力 的培养。当学生遇到实际应用问题时, 往往不知如何下手。在传统的运筹学 实验教学当中, 老师带领学生进行运筹学的实验活动, 对学生的实际情况和 需求考虑较少, 缺乏互动, 学生积极性调动不起来 [3]。

3.3. 传统运筹学实验考核方式多采用实验报告的形式, 老师公布一些传 
统的案例, 学生在实验室运用 Matlab 或 LINGO 等软件进行程序化操作。实 验的时间多是 2 个小时左右, 学生只会机械地套用课本上说的或者老师教的 操作流程进行实验, 老师最后看实验报告情况给出相应的成绩。这种考核模 式无法衡量学生的解决实际问题的能力, 协作能力, 沟通能力[4]。

\section{4. 运筹学实验教学改革探究}

近几年涌现出来的 MOOC (慕课)将为这些问题的解决注入新理念、新思 路、新方法、新经验。针对传统运筹学实验教学面临的三大问题, 我们试图 利用慕课的优点来解决运筹学实验教学的不足, 在混合式课堂教学的基础上 开展教学模式, 教学内容, 考核方式三个方面进行改革。

4.1. 针对问题 3.1, 慕课引发了运筹学理论教学和实验教学理念与方法的 重大变革, 慕课背景下运筹学实验教学中, 学生可以随时随地, 随心所欲的 在各种场合, 如在寝室里或家里完成网络在线的慕课学习, 学习不仅仅局限 在 2 个小时和有限的实验室空间; 将高校的专家、企业的优秀管理人员引入 网络, 共同探讨运筹学的实验问题, 交流经验, 这样使学生的学习资源更加 丰富, 获取知识的来源更加开放、自由和多样。同时, 可以加强学校和企业 的良好结合, 使学校中的理论知识、科研成果与企业中的前沿管理相结合, 促进学校和企业的共同发展。

4.2. 针对上节的问题 3.2, 运筹学实验课程的教学目的是培养学生 “优化” 意识, 提高学生决策能力, 建模能力和解决实际问题的能力。运筹学实验教 学不仅仅是理论教学的补充, 更因该是为了培养学生的创新能力而开设的实 践课和实战课。而 MOOC 所具有的开放性恰恰为这个目标的实现提供了一个 新的契机。我们一方面引导学生进行设计性实验, 也就是熟悉完运筹学知识 框架内的不同类型的优化问题调用的 Matlab 的工具箱, 试图修正或者重新编 写程序, 并验证程序的正确性, 让学生掌握建模方法和程序设计; 另一方面 鼓励学生进行综合性实验, 让学生根据自己所感兴趣的社会经济活动的某方 面, 也可以通过网络课堂与企业交流, 就企业遇到的实际问题进行数学建模, 自编程序得到结果, 改变演示型和验证型实验的不足, 使学生更深刻理解运 筹学的具体功能及使用的方法和意义, 进而实现学校培养学生的目标, 为建 立独立的实验教学方法体系铺平的道路。整个实验过程中以学生为中心、以 教师为辅。学生可以在网络中留言, 说明自己的需要和自己在运筹学实验中 遇到的问题和认为不合理的地方, 以便在以学生为主体的情况下大家共同不 断改进 MOOC 的运筹学实验教学, 使教学更加人性化和个性化, 提高学生的 学习兴趣和求知欲, 满足学生或企业人员的实际需要。

4.3. 针对上节提到的问题 3.3, 教师组织若干高质量设计性实验和综合性 实验训练, 把学生分小组, 教师把这些实验训练分配给各小组, 要求小组内 部分工合作, 每个学习小组选一名小组长, 负责组内人员分工和学习情况交 流汇报。小组成员间可以互相讨论, 网上提交实验结果, 包括所建立的模型、 所用的算法、程序、实验过程中的收获感悟发表等。教师可以在线对运筹学 实验过程和结果进行评价和打分, 这样大大节约了教师的时间, 提高了工作

效率。考核指标可以分为预习结果心得体会、回答问题互帮互助、综合性实 
践训练、作业评价和最后实训表现等多环节过程考核, 课堂讨论占 $15 \%$, 综 合性实践训练占 $30 \%$, 日常小作业占 15\%, 合作互助体现占 $40 \%$ 。考核是指 挥棒, 过程考核就是给学生相应的激励, 但最后的实践训练环节则是学生成 就感的表现。

\section{5. 结论}

总之，通过以上提到的几点慕课背景下的这种全新教学模式改革，能够 大大提高学生的学习效率和效果, 也能培养出团队协作精神, 加固企业和学 校联合的桥梁, 呈现 “未来教育” 的曙光。为学习者提供了极大的便利, 节 约了很多人力和资源, 是运筹学实验教学的发展趋势。这种对学习者学习能 力的培养大大节约了教师的时间，提高了工作效率。

\section{基金项目}

2015 年度上海市运筹学精品课程建设项目, 上海理工大学 2017 年教学 改革项目。

\section{References}

[1] 黄震. 慕课及其给上海教育带来的机遇与挑战[J]. 世界科学, 2014(3): 22-22.

[2] 李继超. 《运筹学》教学方法的改革与实践 [J]. 价值工程, 2014(15): 235-236.

[3] 杨茂盛, 孔凡楼, 张炜. 对运筹学课程教学改革的看法和建议 [J]. 西安建筑科技 大学学报: 社会科学版, 2006, 25(4): 108-110.

[4] 丁妍, 王颖. “慕课”背景下我国大学教师的教学应对[J]. 中国高等教育, 2014(20): 59-60. 